\section{Film compression bandage: a new modality to improve sclerotherapy of superficial varicosities}

\author{
Johann Chris Ragg \\ angioclinic ${ }^{\circledR}$ vein centers, Berlin, \\ Germany
}

\section{Introduction}

Therapy for varicose veins and venous insufficiency currently undergoes a tremendous change as surgery is step by step replaced by endovenous methods. However, superficial varicosities of considerable size still are treated by phlebectomy, as simple foam sclerotherapy with Cabrera-type foams is frequently leading to symptomatic inflammations, visible residuals or ugly stainings, in spite of compression protocols according to guidelines and recommendations. Sclerofoam will lead to target vein occlusion, but thrombus formation is required to obtain permanent obliteration. Thrombus is organized and resorbed in an inflammatory procedure over weeks, while wall inflammation generates transmural vessel connections and thus a second-phase blood entry to a vein which is actually meant to vanish. Symptoms are the more evident the closer the varicose vein is to the skin and its multitude of sensitive nerves. If attempts are made to reduce such unwanted sequelae by mini-thrombectomies, practically expression or suction of thrombus from varices in local anesthesia, this is usually more painful for the patients than the whole initial treatment. As endovenous therapy can be performed pain free at high levels of comfort with immediate ambulation and no further restrictions, a more consequent compression like 4-6 weeks with bandages day and night are today no longer communicable. Eccentric compression can partially solve clinical vein regression problems, but require additional efforts and continuity is limited. To approach the beneficial influence of a continuous compression and to establish a minimum of blood clot constantly for several weeks until inflammation comes to an end, a compression film bandage was developed consisting of an approved polymer film basis with hypoallergenic acrylic glue (3M Inc.) and supportive layers to handle the application as a bandage (Venartis Inc.). The film is placed once and may stay for two to four weeks. After a first pilot study with different films now a second series of cases was performed, including a more detailed diversifi- cation of subgroups. Main endpoints were the frequency of symptomatic inflammatory reactions and wearing comfort. ${ }^{1-11}$

\section{Materials and Methods}

354 patients (23-74 yr/o) with a total of 450 eligible legs with superficial varicosities, 5-16 mm Ø, mean $7.9 \mathrm{~mm} \varnothing$, were included in the study. Cases were randomized to 6 groups with equal diameter distribution $(+/-0.3 \mathrm{~mm})$ : Film for 14 or 28 days with a renewal after $14 \mathrm{~d}$, film plus compression stocking German class 2 for 14 or 28 days day over, and compression stocking alone day over for 14 or 28 days. Prototypes of the elastic compression film bandage
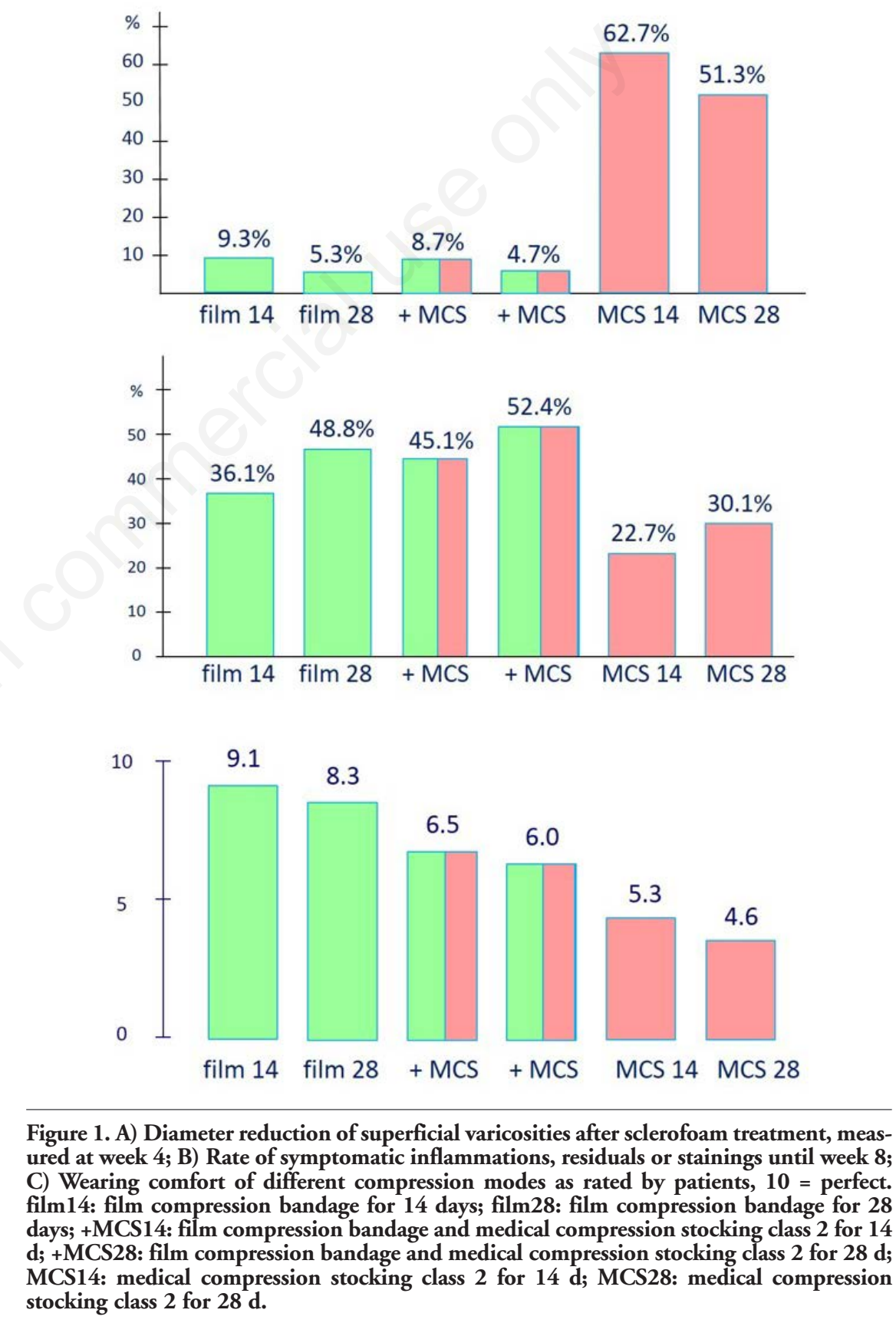

Figure 1. A) Diameter reduction of superficial varicosities after sclerofoam treatment, measured at week 4; B) Rate of symptomatic inflammations, residuals or stainings until week 8; C) Wearing comfort of different compression modes as rated by patients, $10=$ perfect. film14: film compression bandage for 14 days; film 28: film compression bandage for 28 days; +MCS14: film compression bandage and medical compression stocking class 2 for 14 d; +MCS28: film compression bandage and medical compression stocking class 2 for $28 \mathrm{~d}$; MCS14: medical compression stocking class 2 for 14 d; MCS28: medical compression stocking class 2 for $28 \mathrm{~d}$.

[Veins and Lymphatics 2017; 6:6635]

Correspondence: Johann Chris Ragg, angio-

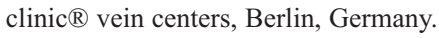

E-mail: ragg@angioclinic.de

Work group: www.venartis.org

This work is licensed under a Creative Commons Attribution 4.0 License (by-nc 4.0).

CC Copyright J. Chris Ragg, 2017

Licensee PAGEPress, Italy

Veins and Lymphatics 2017; 6:6635

$(\mathrm{d}<20 \mu \mathrm{m})$ were applied with $1-1.5 \mathrm{~cm}$ overlapping, covering the target varicosities, immediately after foam sclerotherapy (Aethoxysklerol 1\%, 1+4 with filtrated room air, butterfly $23-25 \mathrm{G}$ if $<6 \mathrm{~mm} \varnothing$, 
micro catheter $16-18 \mathrm{G}$ if $>6 \mathrm{~mm} \varnothing$, PhleboCath 2.0-2.3 for saphenous veins) and worn continuously. Any work, sports and daily showers were allowed. For the first $24 \mathrm{~h}$, a short-stretch bandage was put on top. Follow-up examinations including ultrasound and photography were performed after 2, 4 and 8 weeks.

\section{Results}

All target veins showed total closure at the first control. At the end of compression media application (28 d) vein diameters were reduced by $19.5-67.8 \%$, while variations were depending on the modality (mean values displayed in Figure 1A), but also on the degree of native vein bulging. Combining film bandage with compression stockings was slightly more effective than film bandage alone. Prolonged application periods $(28 \mathrm{~d})$ were more effective than short periods $(14 \mathrm{~d})$ for all modalities.

Symptomatic inflammations of any degree, visible or palpable indurations, or discolorations were observed within 8 weeks (considered to cover $>95 \%$ of such complications) in a maximum of $62.5 \%$, related to cases receiving stocking treatment for $14 \mathrm{~d}$, and $51.3 \%$ if stockings were worn for 28 days (Figure 1B). The use of the film compression bandage reduced inflammatory symptoms to $4.7-9.3 \%$ of the cases. This difference was statistically highly significant $(\mathrm{P}<0.01)$. The need for minithrombectomies was reduced in the same

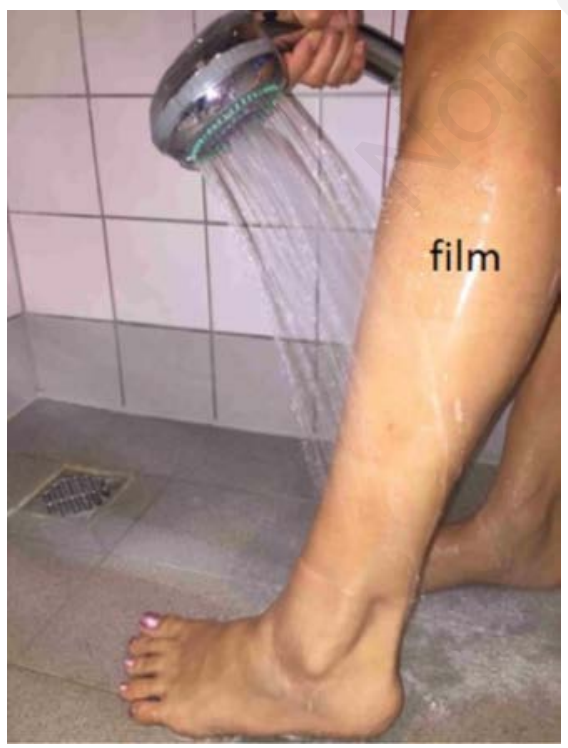

Figure 2. As the film bandage is very thin, invisible and waterproof it allows any kind of work, sports, clothing and even showers.
range.Comfort was rated by the patients according to a 10-degree scale in which 10 was best, defined as no perception of the medium during any activities or at rest. Film bandage for 14 days was the most comfortable modality (9.1) while compression stocking for 28 days was worst (4.6) (Figure 1C). Film bandage combined with compression stocking was more comfortable than compression stocking alone. As a rule, shorter compression periods (14 d) were better accepted than longer periods (28 d). More than $90 \%$ of the patients took showers at more than 4 days a week. The additional comfort of allowing showers, any kind of sports, any kind of clothing and even beach life in summer was greatly appreciated by the patients (Figure 2).

There were no relevant film-related

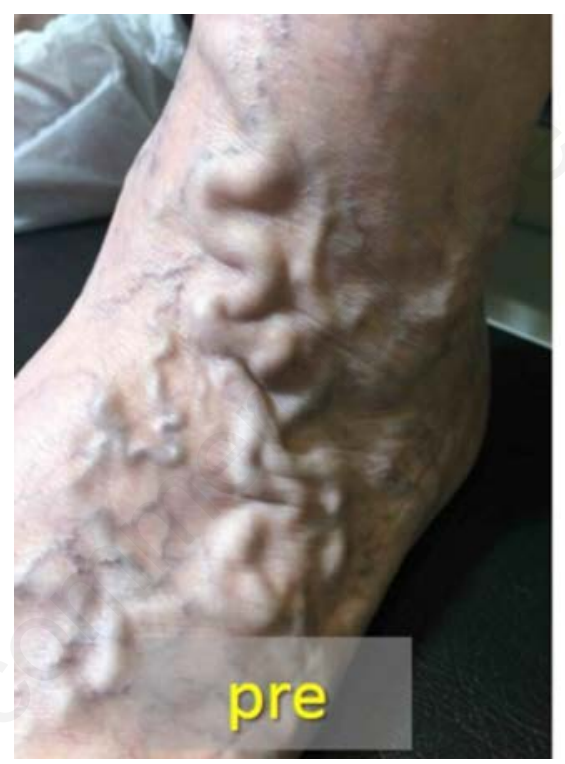

Figure 3. Foot varicosity before and $15 \mathrm{~min}$. after sclerofoam treatment with a film bandage in place. Primary and long-term results have a similar appearance.

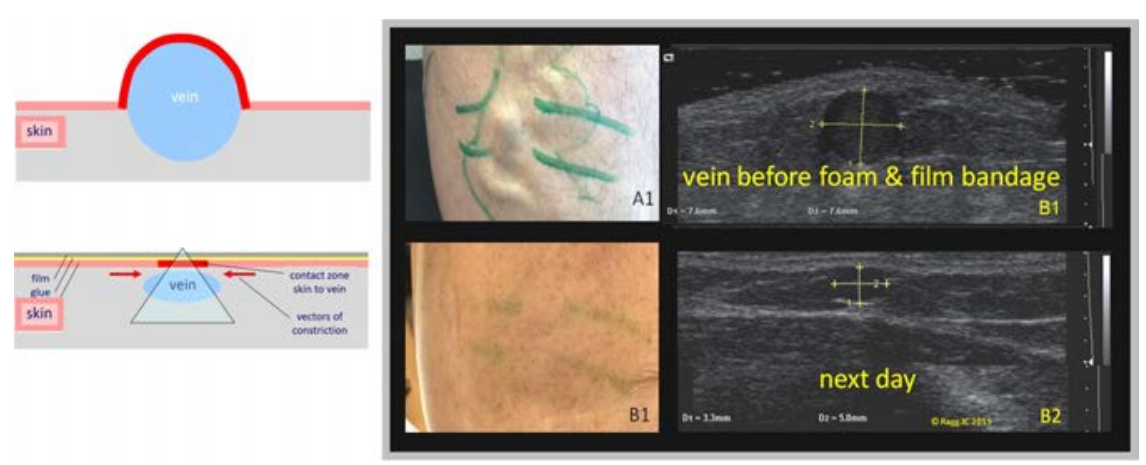

Figure 4. Scheme, visual aspect and ultrasound of a bulging varice before and one day after sclerofoam and film bandage.

[Veins and Lymphatics 2017; 6:6635]

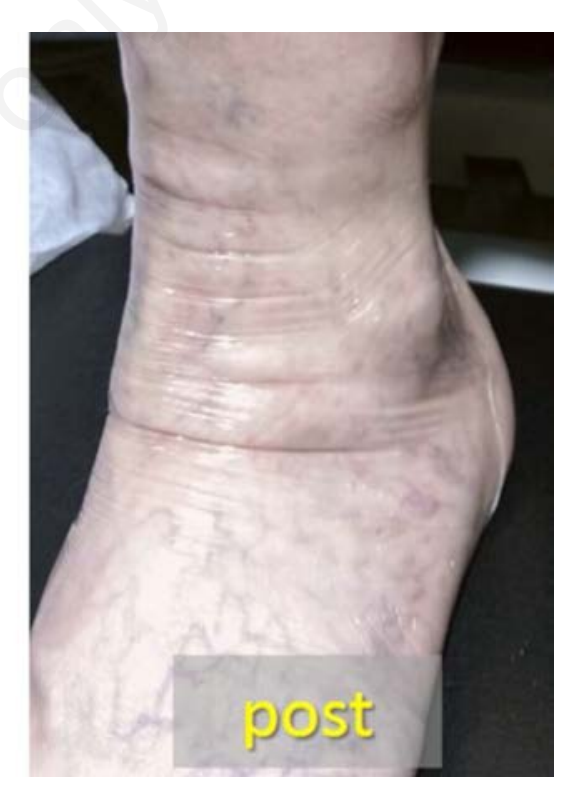

complications: 13/300 film bandage cases showed minor skin reddening, usually near the upper margin. One case showed largearea skin reddening (14 d film only group), allergy could be excluded. Marginal dissolution for $1-2 \mathrm{~cm}$, again in a majority related to the upper margin, was seen in $18.2 \%$ of the cases. A few bandages had to be renewed prior to schedule $(9.2 \%)$.

\section{Discussion}

After the first pilot study ${ }^{10}$ it was not surprising how well the film bandage performed (Figure 3). One particular aspect was the enormous reduction of inflammatory symptoms and staining when using the film bandage, while the diameter reduction was less impressive (Figure 1A and B). 
Different to common textile bandages or stockings there are significant local effects mediated by the glue: The film will form a circumferential functional unit with the skin, adding the elasticity of both components. Furthermore, the unit of glue and skin will locally limit the space available for the vein when it is re-expanding after the end of spasm, to a much bigger extent than a non-gluing material can provide. This effect is the biggest at skin level and lost with increasing depth (triangle of constriction). The varicose vein shows a position shifted towards the fatty tissue, reducing the contact area to sensitive skin (Figure 4). These mechanisms may explain why inflammations are less perceived and less seen even when they are detectable in ultrasound. Because of these physical issues, the effects of the film bandage are the better the more a native varicose vein is bulging above skin level. Inflammatory sequelae were more frequent in the group receiving stocking only than in the average of literature reports, $, 2,5,11,12$ which may be due to the large diameters included in this study.

Except from cases which will not tolerate to wear a compression stocking, it seems to be very useful to combine the film with a graduated stocking: stockings, if tailored correctly, will ideally cover the foot to prevent edema while the film is tricky to place circumferentially at the foot, it also might suffer from mechanical stress and moisture in this location. On the other locations, the film improves the wearing comfort of stockings, while the stockings gradually contribute to the symptomatic result of the treatment. Furthermore, it is a great chance to get patients acquainted to (prophylactic) compression stockings for their daily life. After sclerofoam treatment, the type of stocking is chosen according to the patient's general condition according to current guidelines. ${ }^{6,8}$ It will have to be studied if stockings could be class 1 instead of class 2 for $\mathrm{C} 2$ stages, and if additional bandages for the initial 48-72 hours could further improve the results.

It is not yet clear which cases would benefit the most from a prolonged film bandage wearing time (28 d), but vein diameters above $8 \mathrm{~mm}$ and a doubtful qual- ity of foam sclerotherapy (incidental mixture with blood, incomplete blood replacement) seem to be good candidates.

Considering the clinical and esthetic results, there is a new perspective for the treatment of superficial varicosities: there is hardly a reason to perform phlebectomy anymore, not even in very large varices, because foam and film performs very well. Exceptions may be given for varices containing large loads of thrombus, when allergies to acrylic glue may be expected or - not yet evaluated - in very humid and hot climates. Furthermore, very large varicosities at the thigh of exceedingly obese patients could remain subject to phlebectomy, as effect and fixing of the bandage might reach its limits. Once a commercial product is available, foam and film should be preferred in daily practice because no anesthesia is needed and, apart from punctures, no wounds are generated, no risk of infections, bleedings or nerve lesions. Supplementary foam injections, if considered for reimbursement issues, should be scheduled in order to renew the bandage at the same time (e.g. 14 d). In summary, for the first time treatments of epifascial insufficiency can be performed totally by endovenous methods. This statement is not meant as disrespect of precious vascular surgery, but in favor of the patient's safety and comfort.

\section{Conclusions}

The adhesive compression film bandage significantly improves vein regression of foam-treated superficial varicosities $(\mathrm{P}<0.01)$. It prevents symptomatic inflammations and stainings and provides a most comfortable compression. It may be used combined with compression stockings or even as stand-alone modality. There are no limitations in daily work, sports, showers or social life.

\section{References}

1. Fegan WG. Continuous compression technique of injecting varicose veins. Lancet 1963;2:109.

2. Guex JJ, Allaert FA, Gillet JL. Immediate and mid-term complications of sclerotherapy: Report of a prospective multicenter registry of 12,173 sclerotherapy sessions. Dermatol Surg 2005;31:123-8.

3. Mosti G. Post-treatment compression: duration and techniques. Phlebology 2013;28:21-4.

4. Nootheti PK, Cadag KM, Magpantay A, Goldman MP. Efficacy of graduated compression stockings for an additional 3 weeks after sclerotherapy treatment of reticular and telangiectatic leg veins. Dermatol Surg 2009;35:53.

5. O'Hare JL, Stephens J, Parkin D, Earnshaw JJ. Randomized clinical trial of different bandage regimens after foam sclerotherapy for varicose veins. Br J Surg 2010;97:650.

6. Partsch H. Evidence based compression therapy. VASA 2007;34:1-39.

7. Partsch H, Mosti G, Uhl JF. Unexpected venous diameter reduction by compression stocking of deep, but not of superficial veins. Veins and Lymphatics 2012;1:e3.

8. Rabe E, Breu F, Cavezzi A, et al. European guidelines for sclerotherapy in chronic venous disorders. Phlebology 2014;29:338-54.

9. Ragg JC. Eccentric compression of large varicose veins after foam sclerotherapy using a novel silione gel pad. Phlebologie 2014;43:250-6.

10. Ragg JC. Compression film bandage: a new modality providing continuous and sports-suitable vein compression after foam sclerotherapy. Phlebologie 2015;44:249-55.

11. Scurr JH, Coleridge-Smith P, Cutting P. Varicose veins: optimum compression following sclerotherapy. Ann R Coll Surg Engl 1985;67:109-11.

12. Weiss RA, Sadick NS, Goldman MP, Weiss MA. Post-sclerotherapy compression: controlled comparative study of duration of compression and its effects on clinical outcome. Dermatol Surg 1999;25:105-8. 